\title{
LINEARLY CONTROLLED ASYMPTOTIC DIMENSION OF THE FUNDAMENTAL GROUP OF A GRAPH-MANIFOLD
}

\author{
A. SMIRNOV
}

\begin{abstract}
We prove the estimate $\ell$-asdim $\pi_{1}(M) \leq 7$ for the linearly controlled asymptotic dimension of the fundamental group of any 3-dimensional graph-manifold $M$. As applications, we show that the universal cover $\widetilde{M}$ of $M$ is an absolute Lipschitz retract and admits a quasisymmetric embedding into the product of 8 metric trees.
\end{abstract}

\section{$\S 1$. INTRODUCTION}

A motivation of the present work is a result of Bell and Dranishnikov [3, Theorem 1'] that the asymptotic dimension of a graph-group whose vertex groups have finite asymptotic dimensions is also finite. The fundamental groups of graph-manifolds are graphgroups. However, it is unclear whether there exists an analog of the Bell-Dranishnikov result for the linearly controlled asymptotic dimension. A construction of suitable coverings for the universal cover of a graph-manifold, which we present below, allows us to prove the following statement.

Theorem 1. For the fundamental group of a graph-manifold $M$, taken with any word metric, and for its linearly controlled asymptotic dimension, we have $\ell$-asdim $\pi_{1}(M) \leq 7$.

Corollary 1. Suppose $\widetilde{M}$ is the universal cover of a graph-manifold $M$. Then we have $3 \leq \operatorname{dim}_{\mathrm{AN}} \widetilde{M} \leq 7$, where $\operatorname{dim}_{\mathrm{AN}}$ is the Assouad-Nagata dimension, $M$ being taken with any Riemannian metric and $\widetilde{M}$ with the metric lifted from $M$.

The next two corollaries are obtained as applications of Corollary 1 and 9, Theorem 1.3, Theorem 1.5].

Corollary 2. Let $\widetilde{M}$ be the universal cover of a graph-manifold $M$ taken with the Riemannian distance $d$ lifted from $M$. Then for all sufficiently small $p \in(0,1)$, there exists a bi-Lipschitz embedding of $\left(\widetilde{M}, d^{p}\right)$ into the product of 8 metric trees; in particular, $(\widetilde{M}, d)$ admits a quasisymmetric embedding into the product of 8 metric trees.

Corollary 3. The universal cover $\widetilde{M}$ of a graph-manifold is an absolute Lipschitz retract; i.e., given a metric space $X$, there is $C>0$ such that for every subset $A \subset X$ and every $\lambda$-Lipschitz map $f: A \rightarrow \widetilde{M}, \lambda>0$, there exists a $C \lambda$-Lipschitz extension $\bar{f}: X \rightarrow \widetilde{M}$ of $f$.

\section{§2. Preliminaries}

In this section we recall some basic definitions and notation. Let $X$ be a metric space. We denote by $|x y|$ the distance between $x, y \in X$, and $d(U, V):=\inf \{|u v|: u \in U, v \in V\}$ is the distance between $U, V \subset X$. We write $B_{r}(x)=\left\{x^{\prime} \in X:\left|x x^{\prime}\right| \leq r\right\}$ for the ball

2010 Mathematics Subject Classification. Primary 57M50, 55M10; Secondary 05C05, 20F69.

Key words and phrases. Graph-manifold, asymptotic dimension. 
with center $x$ and radius $r$. A map $f: X \rightarrow Y$ is said to be quasi-isometric if there exist $\lambda \geq 1, C \geq 0$ such that

$$
\frac{1}{\lambda}|x y|-C \leq|f(x) f(y)| \leq \lambda|x y|+C
$$

for each $x, y \in X$. Metric spaces $X$ and $Y$ are quasi-isometric if there is a quasi-isometric map $f: X \rightarrow Y$ such that $f(X)$ is a net in $Y$. In this case $f$ is called a quasi-isometry.

We say that a family $\mathcal{U}$ of subsets of $X$ is a covering if for each point $x \in X$ there is a subset $U \in \mathcal{U}$ such that $x \in U$. A family $\mathcal{U}$ of sets is disjoint if each two sets $U, V \in \mathcal{U}$ are disjoint. The union $\mathcal{U}=\bigcup\left\{\mathcal{U}^{\alpha}: \alpha \in \mathcal{A}\right\}$ of disjoint families $U^{\alpha}$ is said to be $n$-colored, where $n=|\mathcal{A}|$ is the cardinality of $\mathcal{A}$.

Also recall that a family $\mathcal{U}$ is $D$-bounded if the diameter of every $U \in \mathcal{U}$ does not exceed $D$, $\operatorname{diam} U \leq D$. An $n$-colored family of sets $\mathcal{U}$ is $r$-disjoint if for every color $\alpha \in \mathcal{A}$ and each two sets $U, V \in \mathcal{U}^{\alpha}$ we have $d(U, V) \geq r$.

The linearly controlled asymptotic dimension is a version of Gromov's asymptotic dimension asdim.

Definition 1 (Roe [10). The linearly controlled asymptotic dimension of a metric space $X, \ell$-asdim $X$, is the smallest integer $n$ such that for any sufficiently large real $R$ there exists an $(n+1)$-colored, $R$-disjoint, and $C R$-bounded covering of the space $X$, where the number $C>0$ is independent of $R$.

A tripod in a geodesic metric space $X$ is a union of three geodesic segments $x t \cup y t \cup z t$ that have only one common point $t$. We say that $t$ is the center of the tripod. A geodesic metric space $X$ is called a metric tree if each triangle in it is a tripod (possibly, degenerate).

Let $T$ be a metric tree. We recall the well-known construction of a 2-colored, $r$-disjoint, $3 r$-bounded covering of $T$ (see [10). Fix some vertex $v \in T$. Suppose $k \geq 1$ is an integer and consider the annuli

$$
A_{k}:=\{x \in T: k r \leq|v x| \leq(k+1) r\}
$$

of width $r$ centered at $v$. For $x, y \in A_{k}$, we say that $x \sim_{k} y$ if $|z v| \geq\left(k-\frac{1}{2}\right) r$, where $z$ is the center of the tripod with vertices $x, y, v$. It is easily checked (see [10]) that $\sim_{k}$ is an equivalence relation on $A_{k}$. We shall use the following 2-colored covering: $\mathcal{U}:=\mathcal{U}^{1} \cup \mathcal{U}^{2}$, where $\mathcal{U}^{1}$ consists of the ball $B_{r}(v)$ and the equivalence classes for $\sim_{k}$ corresponding to all even $k$. The family $\mathcal{U}^{2}$ consists of the equivalence classes for $\sim_{k}$ corresponding to all odd $k$. In $[10$ it was shown that this covering is $r$-disjoint and $3 r$-bounded. We call it the standard $(r, v)$-covering of $T$.

We also use a modified covering, where the ball $B_{r}(v)$ is replaced by the ball $B_{d}(v)$ with $0<d \leq r$. The annuli $A_{k}$ are replaced by the annuli

$$
B_{k}:=\{x \in T:(k-1) r+d \leq|v x| \leq k r+d\}
$$

of width $r$ and centered at $v$. Now, for each integer $k>1$, and also for $k=1$ and $d \geq r / 2$, on $B_{k}$ we introduce the following equivalence relation: $x \sim_{k} y$ if and only if $|z v| \geq\left(k-\frac{3}{2}\right) r+d$. If $k=1$ and $d<r / 2$, then we say that all points in $B_{1}$ are $\sim_{1}$-equivalent. As before, it is easy to check that the modified covering is $r$-disjoint and $3 r$-bounded. We call this covering the standard $(r, d, v)$-covering of $T$.

Let $G$ be a finitely generated group and $S \subset G$ a finite symmetric generating set for $G$ $\left(S^{-1}=S\right.$ ). Recall that a word metric on the group $G$ (with respect to $S$ ) is the metric determined by the norm $\|\cdot\|_{S}$, where for each $g \in G$ its norm $\|g\|_{S}$ is the smallest number of elements of $S$ whose product is $g$. In this paper we consider only finitely generated groups with a word metric. 
Definition 2. By a graph-manifold we mean a closed three-dimensional manifold that admits a finite cover by a connected orientable manifold glued from blocks along boundary tori in a regular way. By a block we mean a trivial $S^{1}$-fibration over a surface with negative Euler characteristic and nonempty boundary. A gluing of two blocks $M_{1}$ and $M_{2}$ (possibly, $M_{1}=M_{2}$ ) along boundary tori $T_{1} \subset \partial M_{1}$ and $T_{2} \subset \partial M_{2}$ is said to be regular if the $S^{1}$-fibers coming up from $T_{1}$ and $T_{2}$ are not homotopic on the glued torus.

Fix a Riemannian metric on a graph-manifold $M$. The following fact is well known [5].

Lemma 1. Let $Y$ be a compact metric space with length metric. Let $X$ be a universal cover of $Y$ with the metric lifted from $Y$. Then $X$ is quasi-isometric to the group $\pi_{1}(Y)$ with any word metric.

\section{§3. Graph-Manifold MOdel}

3.1. Description of the model. Consider the hyperbolic plane $\mathbb{H}_{\kappa}^{2}$ with a curvature $-\kappa(\kappa>0)$ such that the side of a rectangular equilateral hexagon $\theta$ in the plane $\mathbb{H}_{\kappa}^{2}$ has length 1 . Let $\rho$ be the distance between the middle points of sides that have a common adjacent side, and let $\delta$ be the diameter of $\theta$. It is clear that $\rho \leq 2$ and $\delta \leq 3$.

We mark each second side of $\theta$ (three sides are marked) and consider a set $\mathrm{H}_{0}$ defined as follows. Take the subgroup $G_{\theta}$ of the isometry group of $\mathbb{H}_{\kappa}^{2}$ generated by reflections in (three) marked sides of $\theta$, and let $\mathrm{H}_{0}$ be the orbit of $\theta$ with respect to $G_{\theta}$. Then $\mathrm{H}_{0}$ is a convex subset in $\mathbb{H}_{\kappa}^{2}$ divided into hexagons that are isometric to $\theta$. Furthermore, the boundary of $\mathrm{H}_{0}$ has infinitely many connected components each of which is a geodesic line in $\mathbb{H}_{\kappa}^{2}$.

The graph $T_{0}$ dual to the decomposition of $\mathrm{H}_{0}$ into hexagons is the standard binary tree whose vertices are all of degree three. Any metric space isometric to $\mathrm{H}_{0}$ will be called a $\theta$-tree. Given a vertex $p$ of $T_{0}$, we denote by $\theta_{p}$ the corresponding hexagon in $\mathrm{H}_{0}$.

Every boundary line $\ell$ of $\mathrm{H}_{0}$ is the union of segments of length 1 , each of which is a side of a hexagon in $\mathrm{H}_{0}$. Thus, $\ell$ determines a sequence of vertices of $T_{0}$ connected consecutively by edges that form a line $\bar{\ell}$ in $T_{0}$.

Consider the line $\mathbb{R}$ and its partition into segments $\mathbb{R}=\bigcup\{[i, i+1]: i \in \mathbb{Z}\}$. Then the graph $S_{0}$ dual to this partition is an infinite tree with all vertices having degree 2; in particular, $S_{0}$ is homeomorphic to $\mathbb{R}$. Given a vertex $q$ of $S_{0}$, we denote by $I_{q} \subset \mathrm{H}_{0}$ the corresponding segment.

Remark 1. In what follows, we shall view $T_{0}$ and $S_{0}$ as metric spaces with metrics such that the length of each edge is equal to 1 . Then these metric spaces are metric trees. We denote the set of vertices in $T_{0}$ by $\mathrm{V}\left(T_{0}\right)$, and the set of vertices in $S_{0}$ by $\mathrm{V}\left(S_{0}\right)$.

Remark 2. Given distinct vertices $p, q \in \mathrm{V}\left(T_{0}\right)$ in $T_{0}$ and $x \in \theta_{p}, y \in \theta_{q}$, we have

$$
|p q|-1 \leq|x y| \leq(|p q|-1) \rho+2 \delta .
$$

Similarly, given distinct $p, q \in \mathrm{V}\left(S_{0}\right)$ and $x \in I_{p}, y \in I_{q}$, we have

$$
|p q|-1 \leq|x y| \leq|p q|+1 .
$$

Remark 3. Note that, for each connected subset $U \subset T_{0}$, the set $U^{\prime}:=\bigcup\left\{\theta_{p}: p \in\right.$ $\left.U \cap \mathrm{V}\left(T_{0}\right)\right\}$ is convex. Similarly, for each connected subset $V \subset S_{0}$, the set $V^{\prime}:=$ $\bigcup\left\{I_{q}: q \in V \cap \mathrm{V}\left(S_{0}\right)\right\}$ is convex.

Now, we describe the following metric space $X$. 
Construction. Let $T$ be an infinite tree, all vertices of which have countably infinite degree. Denote by $\mathrm{V}(T)$ the set of all vertices of $T$ and by $\mathrm{E}(T)$ the set of all edges of $T$. For each $v \in \mathrm{V}(T)$, we consider the metric product $X_{v}:=\mathrm{H}_{0} \times \mathbb{R}$ and fix a bijection $b_{v}$ between the set $\mathrm{E}_{v}$ of all edges adjacent to $v$ and all the boundary lines in the factor $\mathrm{H}_{0}$. Note that the partition of $\mathrm{H}_{0}$ into hexagons and the partition of $\mathbb{R}$ into segments give rise to a partition of $X_{v}$ into sets isometric to $\theta \times[0,1]$; we call such sets bricks. Furthermore, each boundary plane $\sigma \subset X_{v}$ is obtained as the product of $\mathbb{R}$ and a boundary line $\ell$ in the $\theta$-tree factor, and the partition of $X_{v}$ into bricks gives rise to a partition of $\sigma$ into squares with side length 1 , to be called the grid on the plane $\sigma$. Next, for each edge $u v$ in $\mathrm{E}(T)$, we consider the boundary planes $\sigma_{u}=b_{u}(u v) \times \mathbb{R}_{u} \subset X_{u}$ and $\sigma_{v}=b_{v}(u v) \times \mathbb{R}_{v} \subset X_{v}$, respectively, and glue them by an isometry that glues the grids on $\sigma_{u}$ and $\sigma_{v}$, flipping the factors $\mathbb{R}_{u}, \mathbb{R}_{v}$ to the factors $b_{v}(u v), b_{u}(u v)$, respectively. We call the resulting space the model and denote it by $X$.

Recall that a complete geodesic metric space $Y$ is called a CAT(0)-space if the following is true: for each triple of points $x, y, z \in X$ and a point $t \in y z$, if points $\bar{x}, \bar{y}, \bar{z}$ on the plane $\mathbb{R}^{2}$ are such that $|x y|=|\bar{x} \bar{y}|,|y z|=|\bar{y} \bar{z}|,|x z|=|\bar{x} \bar{z}|$ and the point $\bar{t} \in[\bar{y} \bar{z}]$ satisfies $|y t|=|\bar{y} \bar{t}|$, then $|x t| \leq|\bar{x} \bar{t}|$. A CAT(0)-space is also called a Hadamard space. The spaces $\mathrm{H}_{0}$ and $\mathbb{R}$ are $\mathrm{CAT}(0)$-spaces, so that the $X_{v}, v \in \mathrm{V}(T)$, and $X$ are also CAT(0)-spaces (see, e.g., [5]).

3.2. The model $X$ as the universal cover of a closed graph-manifold. Here we construct a closed graph-manifold $Q$ (with $C^{1}$-smooth Riemannian nonpositively curved metric) whose universal cover $\widetilde{Q}$ is isometric to $X$.

Let $\omega$ be the union of adjacent hexagons $\theta_{1}, \theta_{2} \subset \mathrm{H}_{0}$. Denote by $\tau: \theta_{1} \rightarrow \theta_{2}$ the reflection with respect to the common side $\theta_{1} \cap \theta_{2}$ and consider the surface $P_{0}$ obtained from $\omega$ by gluing the marked sides matched by $\tau$. Then $P_{0}$ is a surface of constant curvature $-\kappa$ with geodesic boundary. The boundary $\partial P_{0}$ consists of 3 components $\gamma_{1}, \gamma_{2}$, and $\gamma_{3}$ of length 2 . The hexagon vertices subdivide every $\gamma_{i}, i=1,2,3$, into a pair of segments of length 1 .

Let us mark the images of the hexagons' vertices (so, there will be 6 marked points). Note that the universal cover of $P_{0}$ is isometric to $\mathrm{H}_{0}$. We consider the circle $S_{2}^{1}$ of length 2 and mark two diametrically opposite points $s_{1}$ and $s_{2}$ on it. For each boundary component $\gamma_{i}(i \in\{1,2,3\})$, consider an isometry $\Phi_{i}: \gamma_{i} \rightarrow S_{2}^{1}$ taking the marked points into the marked points.

Let $P:=P_{0} \times S_{2}^{1}$. Consider an isometric copy $P^{\prime}$ of $P$. All the elements of $P^{\prime}$ will be denoted by the same letters but with a prime. Let $Q$ be the manifold obtained by gluing $P$ and $P^{\prime}$ along the boundary tori $\gamma_{i} \times S_{2}^{1} \subset \partial P$ and $S_{2}^{1} \times \gamma_{i}^{\prime} \subset \partial P^{\prime}$ in accordance with the isometries

$$
\Phi_{i} \times \Phi_{i}^{\prime-1}: \gamma_{i} \times S_{2}^{1} \longrightarrow S_{2}^{1} \times \gamma_{i}^{\prime}, \quad i \in\{1,2,3\} .
$$

Note that $Q$ is a graph-manifold, the universal cover $\widetilde{Q}$ of which consists of blocks isometric to $\mathrm{H}_{0} \times \mathbb{R}$. Any two of these blocks either are disjoint or intersect over a plane covering a torus $\gamma_{i} \times S_{2}^{1}, i \in\{1,2,3\}$. This plane is divided by the lines covering the circles $\left\{x_{i}\right\} \times S_{2}^{1}$ and $\gamma_{i} \times\left\{s_{i}\right\}$ (where $x_{i} \in \gamma_{i}$ and $s_{i} \in S_{2}^{1}$ are marked points) into squares with side 1 . It is clear that $\widetilde{Q}$ is isometric to $X$. We recall the following result [2. Theorem 2.1].

Theorem 2. Any two graph-manifolds have bi-Lipschitz homeomorphic universal covers; in particular, their fundamental groups are quasi-isometric.

This theorem shows that for any graph-manifold $M$ its fundamental group $\pi_{1}(M)$ is quasi-isometric to the fundamental group $\pi_{1}(Q)$ of the manifold $Q$, and, by Lemma 1, 
$\pi_{1}(Q)$ is quasi-isometric to the model $X$. Therefore, since $\ell$-asdim is a quasi-isometry invariant (see, e.g., [9]), we have $\ell$-asdim $\pi_{1}(M)=\ell$-asdim $X$. So, to prove Theorem 2 it suffices to show that $\ell$-asdim $X \leq 7$. For this, for each $R \in \mathbb{N}, R>10$, we construct an $R$-disjoint, $C R$-bounded, 8-colored covering of the model $X$, where $C=88$.

\section{$\S 4$. Coverings of The MOdeL}

We briefly describe the construction of the required covering of $X$. We fix $R \in \mathbb{N}$, $R>10$. First, for every block $X_{u}, u \in \mathrm{V}(T)$, we construct a 4-colored, $2 R$-disjoint, and $15 R$-bounded covering $\mathcal{W}_{u}$ that is the product of standard 2-colored coverings of the factors in the decomposition $X_{u}=\mathrm{H}_{0} \times \mathbb{R}$. For different neighboring blocks, these coverings are compatible (see Lemma 2) and, taken together, form a 4-colored covering $\widetilde{\mathcal{W}}$ of $X$. The covering $\widetilde{\mathcal{W}}$ is $R$-disjoint (see Proposition 1 below), and this is the main result of the current section. However, all the members of $\widetilde{\mathcal{W}}$ have infinite diameter. Fortunately, every member $W$ of $\widetilde{\mathcal{W}}$ has a tree-like structure, and in Subsection 5.2 we shall construct a 2-colored covering of $W$ similar to the standard 2-colored covering of a tree. A typical $W$ is disconnected, and every connected component of it is convex. For technical reasons, it is convenient to start with extending every $W \in \widetilde{\mathcal{W}}$ to a convex $\bar{W} \supset W$, and then to cover $\bar{W}$ by a 2 -colored $X_{W}$. The extension procedure is described in Subsection 5.1. Convexity plays an important role in proving the properties of $x_{W}$. After that, we forget about the extension $\bar{W}$ and restrict $X_{W}$ to $W$. In that way, we produce the required 8-colored covering $X$ of $X$.

4.1. Constructing a covering of a block. Put $N:=2 R+1$. Consider an arbitrary block $X_{v}=\mathrm{H}_{0} \times \mathbb{R} \subset X$. We choose a brick $\theta_{p} \times I_{q} \subset X_{v}$, where $p \in \mathrm{V}\left(T_{0}\right)$ and $q \in \mathrm{V}\left(S_{0}\right)$. Consider the standard $(N, p)$-covering $\mathcal{U}=\mathcal{U}^{1} \cup \mathcal{U}^{2}$ of the tree $T_{0}$ (see $\oiint_{2}$ ). We build a 2-colored covering $\widetilde{\mathcal{U}}=\widetilde{\mathcal{U}}^{1} \cup \widetilde{\mathcal{U}}^{2}$ of the $\theta$-tree as follows: for each set $U \in \mathcal{U}^{i}$, $i \in\{1,2\}$, we define the set

$$
\widetilde{U}:=\bigcup\left\{\theta_{p^{\prime}}: p^{\prime} \in U \cap \mathrm{V}\left(T_{0}\right)\right\}
$$

and consider the families

$$
\tilde{U}^{i}:=\left\{\tilde{U}: U \in \mathcal{U}^{i}\right\}, \quad i \in\{1,2\} .
$$

It is clear that the family $\tilde{\mathcal{U}}$ is a 2-colored covering of the $\theta$-tree. Recall that $\rho$ is the distance between the middle points of two sides of the hexagon $\theta$ that have a common adjacent side, and $\delta$ is the diameter of $\theta$.

Since the covering $U$ is $N$-disjoint and $3 N$-bounded, inequality $(\star)$ implies that the covering $\tilde{\mathcal{U}}$ is $2 R$-disjoint and $((6 R+2) \rho+2 \delta)$-bounded. Since $\rho \leq 2, \delta \leq 3$, and $R>10$, we have $(6 R+2) \rho+2 \delta \leq 13 R$, whence $\widetilde{\mathcal{U}}$ is $2 R$-disjoint and $13 R$-bounded.

Fixing a natural $d \leq N$, we consider the standard $(N, d, q)$-covering $\mathcal{V}=\mathcal{V}^{1} \cup \mathcal{V}^{2}$ of the tree $S_{0}$ (see $\oiint_{2}$ ). We build a 2-colored covering $\widetilde{\mathcal{V}}=\widetilde{\mathcal{V}}^{1} \cup \widetilde{\mathcal{V}}^{2}$ of the line $\mathbb{R}$ as follows: for each set $V \in \mathcal{V}^{i}, i \in\{1,2\}$, we consider the set

$$
\widetilde{V}:=\bigcup\left\{I_{p^{\prime}}: p^{\prime} \in V \cap \mathrm{V}\left(S_{0}\right)\right\}
$$

and the families

$$
\tilde{\mathcal{V}}^{i}:=\left\{\tilde{V}: V \in \mathcal{V}^{i}\right\}, \quad i \in\{1,2\} .
$$

Clearly, the family $\tilde{\mathcal{V}}$ is a 2-colored covering of $\mathbb{R}$. Note that, since the family $\mathcal{V}$ is $N$-disjoint and $3 N$-bounded, inequality $(\star \star)$ implies that the covering $\widetilde{\mathcal{V}}$ is $2 R$-disjoint and $(6 R+2)$-bounded. Since $R>10$, we have $6 R+2 \leq 7 R$, so that the covering $\tilde{\mathcal{V}}$ is $2 R$-disjoint and $7 R$-bounded. 
Consider the 4-colored covering

$$
\mathcal{W}:=\bigcup\left\{\mathcal{W}^{(i, j)}:(i, j) \in\{1,2\} \times\{1,2\}\right\}
$$

of the block $X_{v}$, where

$$
\mathcal{W}^{(i, j)}:=\left\{U \times V: U \in \tilde{\mathcal{U}}^{i}, V \in \tilde{\mathcal{V}}^{j}\right\} .
$$

Since the coverings $\tilde{\mathcal{U}}$ and $\tilde{\mathcal{V}}$ are $2 R$-disjoint, so is the covering $\mathcal{W}$, and since the covering $\widetilde{\mathcal{U}}$ is $13 R$-bounded and $\widetilde{\mathcal{V}}$ is $7 R$-bounded, the covering $\mathcal{W}$ is $R \sqrt{7^{2}+13^{2}}$-bounded, whence it is $15 R$-bounded. This covering of the block $X_{v}$ will be called the $(N, d)$-covering with the initial brick $\theta_{p} \times I_{q}$.

Consider the block $X_{v}$ with the fixed initial brick $\theta_{p_{v}} \times I_{q_{v}}, p_{v} \in \mathrm{V}\left(T_{0}\right), q_{v} \in \mathrm{V}\left(S_{0}\right)$, and its boundary plane $\sigma=\ell \times \mathbb{R}_{v}$, where $\ell$ is a boundary line of the $\theta$-tree factor of $X_{v}$. Let $\bar{\ell}$ be the line in the tree $T_{0}$ that corresponds to $\ell$ (see Subsection 3.1), and let $p^{\prime} \in \bar{\ell}$ be the vertex nearest to $p_{v}$. We denote $n:=\left|p_{v} p^{\prime}\right|$ and put $k \equiv n(\bmod N), 0 \leq k<N$.

Let $X_{u}$ be the block glued to $\sigma$, let $1 \leq d \leq N$ be a natural number, and let $I_{q_{u}} \times \theta_{p_{u}}$ be the brick in $X_{u}$ having the common boundary square with the brick $\theta_{p^{\prime}} \times I_{q_{v}}$. We represent the boundary plane $\sigma$ as the product of the line $\mathbb{R}$ and an appropriate boundary line $\ell^{\prime}$ in the $\theta$-tree factor of the block $X_{u}, \sigma=\mathbb{R}_{u} \times \ell^{\prime}$. Consider the line $\overline{\ell^{\prime}}$ in the tree $T_{0}$ that corresponds to the line $\ell^{\prime}$. Then the vertex $p_{u}$ belongs to $\overline{\ell^{\prime}}$. We choose a vertex $p_{u}^{\prime}$ of $T_{0}$ such that the vertex $p_{u}$ is the point of the line $\bar{\ell}^{\prime}$ nearest to $p_{u}^{\prime}$ and the distance $\left|p_{u} p_{u}^{\prime}\right|$ is equal to $N-d$.

Lemma 2. The $(N, d)$-covering $\mathcal{W}_{v}$ with the initial brick $\theta_{p_{v}} \times I_{q_{v}}$ of the block $X_{v}$ and the $(N, N-k)$-covering $\mathcal{W}_{u}$ with the initial brick $I_{q_{u}} \times \theta_{p_{u}^{\prime}}$ of the block $X_{u}$ agree on the common boundary plane $\sigma$ of the blocks $X_{u}$ and $X_{v}$; i.e., for each pair $(i, j) \in$ $\{1,2\} \times\{1,2\}$, any two sets $V \in \mathcal{W}_{v}^{(i, j)}$ and $U \in \mathcal{W}_{u}^{(j, i)}$ either are disjoint, or their intersections with $\sigma$ coincide.

Proof. Indeed, since the coverings $\mathcal{W}_{v}$ and $\mathcal{W}_{u}$ are products of coverings of the corresponding factors, it suffices to check that the coverings of the factors agree. We have $\sigma=\ell \times \ell^{\prime}$, and the $\mathbb{R}$-factor of the block $X_{u}$ is glued to the line $\ell$. Let $\bar{\ell}$ be the line corresponding to the line $\ell$ in the tree $T_{0}$. If we view the line $\bar{\ell}$ as an isometric copy of the tree $S_{0}$, then the standard $\left(N, p_{v}\right)$-covering of the tree $T_{0}$ induces a covering $\mathfrak{U}^{\prime}$ of the line $\bar{\ell}$, and this is a standard $\left(N, N-k, p^{\prime}\right)$-covering. Therefore, since the side of the hexagon $\theta_{p^{\prime}}$ lying on $\ell$ is glued to the segment $I_{q_{u}}$ of the $\mathbb{R}$-factor of the block $X_{u}$, the standard $\left(N, N-k, q_{u}\right)$-covering of this $\mathbb{R}$-factor coincides with the covering $\mathcal{U}^{\prime}$. The case of the other factors is similar.

4.2. Constructing compatible coverings of blocks. Recall that $T$ is the canonical simplicial tree, all the vertices of which have infinite countable degree. We consider the metric on $T$ such that all the edges have length 1 . We fix an arbitrary vertex $v \in \mathrm{V}(T)$ and call it the root of the tree $T$. We define the level function $l: \mathrm{V}(T) \rightarrow \mathbb{Z}$ by $l\left(v^{\prime}\right):=\left|v v^{\prime}\right|$. The coverings of blocks of the type discussed earlier, which agree with the coverings of the neighboring blocks on the common boundary planes (see Subsection 4.1), will be constructed by induction on the level $m$.

The base of induction : $m=0$.

Let $\theta_{p} \times I_{q}$ be an arbitrary brick in $X_{v}$. Consider the $(N, N)$-covering with the initial brick $\theta_{p} \times I_{q}$ of the block $X_{v}$. We denote this covering by $\mathcal{W}_{v}$.

The induction step: $m \rightarrow m+1$.

Suppose that we have already built the coverings $\mathcal{W}_{v^{\prime}}$ compatible for all blocks $X_{v^{\prime}}$ with $l\left(v^{\prime}\right) \leq m$. Consider an arbitrary vertex $u \in \mathrm{V}(T)$ such that $l(u)=m+1$. The block $X_{u}$ has precisely one boundary plane $\sigma$ along which it is glued with a block $X_{w}$ such that 
$l(w)=m . X_{w}$ is already covered by $\mathcal{W}_{w}$, which is an $\left(N, d_{w}\right)$-covering with the initial brick $\theta_{p_{w}} \times I_{q_{w}}$. Consider the plane $\sigma=\ell \times \mathbb{R}$, the product of a boundary line $\ell$ in the $\theta$-tree and the factor $\mathbb{R}$. Let $\bar{\ell}$ be the line in $T_{0}$ corresponding to $\ell$ (see Subsection 3.1). We take the vertex $p^{\prime}$ in $\bar{\ell}$ that is the nearest to $p_{w}$ and denote $n_{w}:=\left|p_{w} p^{\prime}\right|$. Let $d_{u} \equiv n_{w}$ $(\bmod N), 0 \leq d_{u}<N$. Let $I_{q_{u}} \times \theta_{p_{u}}$ be a brick in $X_{u}$ that has a common boundary square with the brick $\theta_{p^{\prime}} \times I_{q_{w}}$. We represent the boundary plane $\sigma$ as the product of the line $\mathbb{R}$ and a boundary line in the $\theta$-tree factor of the block $X_{u}, \sigma=\mathbb{R} \times \ell^{\prime}$. Consider the line $\overline{\ell^{\prime}}$ in $T_{0}$ that corresponds to the line $\ell^{\prime}$. It is clear that the vertex $p_{u}$ belongs to $\overline{\ell^{\prime}}$. We choose a vertex $p_{u}^{\prime}$ in $T_{0}$ such that the vertex $p_{u}$ is the point nearest to $p_{u}^{\prime}$ on the line $\bar{\ell}^{\prime}$ and the distance $\left|p_{u} p_{u}^{\prime}\right|$ is equal to $N-d_{w}$. Let $\mathcal{W}_{u}$ denote the $\left(N, N-d_{u}\right)$-covering of the block $X_{u}$ with the initial brick $I_{q_{u}} \times \theta_{p_{u}^{\prime}}$. Lemma 2 shows that the coverings $\mathcal{W}_{w}$ and $\mathcal{W}_{u}$ of the blocks $X_{w}$ and $X_{u}$ (respectively) agree on the boundary plane $\sigma . \mathcal{W}_{u}$ is a covering of the type we need; the induction step is completed.

4.3. Constructing a covering of the model. Consider the following 4 families of subsets of the model $X$. For each pair of numbers $(i, j) \in\{1,2\} \times\{1,2\}$, we denote

$$
\mathcal{W}^{(i, j)}:=\left\{U: U \in \mathcal{W}_{u}^{(i, j)}, l(u) \text { is even }\right\} \cup\left\{U: U \in \mathcal{W}_{u}^{(j, i)}, l(u) \text { is odd }\right\} .
$$

Moreover, for each pair $(i, j) \in\{1,2\} \times\{1,2\}$, we consider the following relation $\sim_{(i, j)}$ on the family $\mathcal{W}^{(i, j)}: U \sim_{(i, j)} V$ if and only if there are sets $U_{0}=U, U_{1}, \ldots, U_{n+1}=V$ in the family $\mathcal{W}^{(i, j)}$ such that $U_{j} \cap U_{j+1} \neq \varnothing$ for all $j \in\{0, \ldots, n\}$. Clearly, $\sim_{(i, j)}$ is an equivalence relation.

Proposition 1. Suppose $W, U \in \mathcal{W}^{(i, j)}$ and $d(W, U)<R$. Then $W \sim_{(i, j)} U$.

We need two auxiliary facts (see Lemmas 3 and 4) for the proof of Proposition 1 .

Lemma 3. Given $U \in \mathcal{W}^{(i, j)}, U \subset X_{u}$, assume that a boundary plane $\sigma \subset X_{u}$ meets $U$, $U \cap \sigma \neq \varnothing$, and separates a point $x \in X$ and $U$. Then $d(x, U)=d(x, U \cap \sigma)$.

Proof. Without loss of generality, we assume that $x \notin \sigma$. Then, since the hyperplane $\sigma \subset X$ is convex, the segment $x y$ meets $\sigma$ over a point for every $y \in U, x y \cap \sigma=t$.

Recall that we represent the block $X_{u}$ as the product $X_{u}=\mathrm{H}_{0} \times \mathbb{R}$ and also $U=$ $\widetilde{U}_{\theta} \times \widetilde{U}_{\mathbb{R}}$, where $\widetilde{U}_{\theta}, \widetilde{U}_{\mathbb{R}}$ are members of the appropriate coverings of $\mathrm{H}_{0}$ and $\mathbb{R}$, respectively. Then $t=\left(t_{\theta}, t_{R}\right) \in \ell \times \mathbb{R}=\sigma$, where $\ell \in \mathrm{H}_{0}$ is a boundary line, and $y=\left(y_{\theta}, y_{R}\right)$, where $y_{\theta} \in \widetilde{U}_{\theta}, y_{R} \in \widetilde{U}_{\mathbb{R}}$. For $t^{\prime}=\left(t_{\theta}, y_{R}\right) \in \sigma$, we have

$$
\left|x t^{\prime}\right| \leq|x t|+\left|t t^{\prime}\right| \leq|x t|+|t y|=|x y| .
$$

If $t_{\theta} \in \widetilde{U}_{\theta}$, then $t^{\prime} \in U \cap \sigma$ because $y_{R} \in \widetilde{U}_{R}$, whence $|x y| \geq d(x, U \cap \sigma)$. Thus, in what follows we assume that $t_{\theta} \notin \widetilde{U}_{\theta}$.

Let $\theta_{p} \times I_{q}$ be the initial brick in $\mathcal{W}_{u}^{(i, j)}$. Passing to the tree $T_{0}$, we denote by $U_{0}$ the set of all vertices $q^{\prime}$ in $\mathrm{V}\left(T_{0}\right)$ such that $\theta_{q^{\prime}} \subset \widetilde{U}_{\theta}$. Then, by the construction of $\mathcal{W}_{u}^{(i, j)}, U_{0}$ is the vertex set of a member of the standard $(N, p)$-covering of the tree $T_{0}$. We denote this member by $U^{\prime}$. There is $k \in \mathbb{N} \cup\{0\}$ such that $U^{\prime}$ lies in the $N$-annulus

$$
A_{k}=\left\{q \in T_{0}: k N \leq|q p| \leq(k+1) N\right\}
$$

centered at $p$. We denote by $\bar{\ell}$ the line in $T_{0}$ corresponding to $\ell$ and pick a vertex $p_{0} \in \bar{\ell}$ of $T_{0}$ such that $t_{\theta} \in \theta_{p_{0}}$. There are at most two such vertices, and we assume that $p_{0} \in U^{\prime}$ if $t_{\theta} \in \widetilde{U}_{\theta}$. Then the assumption $t_{\theta} \notin \widetilde{U}_{\theta}$ is equivalent to the fact that $p_{0} \notin U^{\prime}$.

Since $y_{\theta} \in \widetilde{U}_{\theta}$, there is a vertex $p_{y}$ of $T_{0}$ such that $y_{\theta} \in \theta_{p_{y}}$ and $p_{y} \in U^{\prime}$. We show that $\operatorname{dist}\left(p_{0}, \bar{\ell} \cap U^{\prime}\right) \leq\left|p_{0} p_{y}\right|$ in $T_{0}$. A (unique) point $p^{\prime} \in \bar{\ell}$ closest to $p$ splits $\bar{\ell}$ into rays $\bar{\ell}_{i}, i=1,2$. Each of the rays $\bar{\ell}_{1}, \bar{\ell}_{2}$ intersects the annulus $A_{k}$ over a segment, and at least one of them lies in $U^{\prime}$. We assume without loss of generality that $\bar{\ell}_{1} \cap A_{k} \subset U^{\prime}$. 
There is a shortest segment $\bar{\gamma} \subset \bar{\ell}$ between $p_{0}$ and $\bar{\ell} \cap U^{\prime}$. If $p_{0} \in \bar{\ell}_{1}$, then $\bar{\gamma} \subset \bar{\ell}_{1}$ is a shortest path in $T_{0}$ between $p_{0}$ and $U^{\prime}$. Thus, the length $|\bar{\gamma}|$ is equal to $d\left(p_{0}, U^{\prime}\right)$, whence $d\left(p_{0}, \bar{\ell} \cap U^{\prime}\right) \leq\left|p_{0} p_{y}\right|$.

It remains to consider the case where $p_{0} \in \bar{\ell}_{2}$ and $\bar{\ell}_{2}$ is disjoint with $U^{\prime}$ (the latter happens precisely when $k>1$ and $\left.\left|p p^{\prime}\right| \leq\left(k-\frac{1}{2}\right) N\right)$. As above, we have $\left|p^{\prime} p_{y}\right| \geq$ $d\left(p^{\prime}, U^{\prime}\right)=d\left(p^{\prime}, \bar{\ell} \cap U^{\prime}\right)$. On the other hand, any path in $T_{0}$ between $p_{0}$ and $U^{\prime}$ passes through $p^{\prime}$. Therefore, $\left|p_{0} p_{y}\right|=\left|p_{0} p^{\prime}\right|+\left|p^{\prime} p_{y}\right| \geq|\bar{\gamma}|=d\left(p_{0}, \bar{\ell} \cap U^{\prime}\right)$.

The geodesic segment ty $\subset X_{u}$ projects to the geodesic segment $t_{\theta} y_{\theta}$ in the factor $\mathrm{H}_{0}$ of $X_{u}$. While moving from $t_{\theta}$ to $y_{\theta}$ along $t_{\theta} y_{\theta}$, we pass through the segment $p_{0} p_{y} \subset T_{0}$, so that $\left|t_{\theta} y_{\theta}\right| \geq\left|p_{0} p_{y}\right|-1$ by $(\star)$.

Let $\gamma=t^{\prime} z \subset \ell \times y_{R} \subset \sigma$ be the segment between $t^{\prime}$ and $z \in U \cap \sigma$ that corresponds to $\bar{\gamma} \subset \bar{\ell}$. The length of $\gamma$ is at most the number of interior vertices of $\bar{\gamma}$ plus one, $|\gamma| \leq|\bar{\gamma}|-1$. Since $\left|p_{0} p_{y}\right| \geq|\bar{\gamma}|$, we obtain $\left|t_{\theta} y_{\theta}\right| \geq|\gamma|$. It follows that $|t y| \geq|t z|$ because both triangles $t t^{\prime} y$ and $t t^{\prime} z$ are flat with right angles at $t^{\prime}$. Then, moreover, we have $|x y| \geq|x z| \geq d(x, U \cap \sigma)$.

Lemma 4. Suppose $\sigma$ is the common plane of the blocks $X_{u}$ and $X_{u^{\prime}}$ such that $\left|v u^{\prime}\right|<$ $|v u|$, where $v$ is the root of $T$. Assume that $U \in \mathcal{W}^{(i, j)}, U \subset X_{u}, U$ is disjoint with $\sigma$, $U \cap \sigma=\varnothing$. Then $d(U, \sigma) \geq R$.

Proof. Let $\theta_{p} \times I_{q}$ be the initial brick in $\mathcal{W}_{u}^{(i, j)}$. Recall that $U=\widetilde{U}_{\theta} \times \widetilde{U}_{R}$, where $\widetilde{U}_{\theta}$ and $\widetilde{U}_{R}$ are members of the appropriate coverings of the $\theta$-tree $\mathrm{H}_{0}$ and $\mathbb{R}$, respectively. We denote by $U_{0}$ the set of all vertices $q^{\prime} \in \mathrm{V}\left(T_{0}\right)$ such that the hexagon $\theta_{q^{\prime}}$ belongs to $\widetilde{U}_{\theta}$. Then $U_{0}$ is the vertex set of a member $U^{\prime}$ of the standard $(N, p)$-covering of the tree $T_{0}$. There is $k \in \mathbb{N} \cup\{0\}$ such that $U^{\prime}$ is a subset of the $N$-annulus

$$
A_{k}=\left\{q \in T_{0}: k N \leq|q p| \leq(k+1) N\right\}
$$

centered at $p$.

Representing $\sigma=\ell \times \mathbb{R}$, where $\ell \subset \mathrm{H}_{0}$ is an appropriate boundary line, we let $\bar{\ell} \subset T_{0}$ be the line corresponding to $\ell$. Our assumption on $\sigma$ and the construction of the family $\mathcal{W}^{(i, j)}$ imply that $d(p, \bar{\ell}) \leq N$, and thus, $\bar{\ell}$ meets every annulus $A_{k}, k \geq 0$. By assumption, $\ell$ is disjoint with $\widetilde{U}_{\theta}$, whence $\widetilde{U}_{\theta}$ is disjoint with any hexagon of $\mathrm{H}_{0}$ that meets $\ell$. Thus, $\bar{\ell}$ is disjoint with $U^{\prime}$. Since $\bar{\ell} \cap A_{k} \neq \varnothing$ is covered by members of the standard $(N, p)$ covering of $T_{0}$ having the same color $i \in\{1,2\}$ as $U^{\prime}$, and the covering is $N$-disjoint, we have $d\left(U^{\prime}, \bar{\ell} \cap A_{k}\right) \geq N$. Therefore, $d\left(U^{\prime}, \bar{\ell}\right) \geq N / 2$. Moreover, since $N=2 R+1$ is odd and the distance $d\left(U^{\prime}, \bar{\ell}\right)$ is an integer, we actually have $d\left(U^{\prime}, \bar{\ell}\right) \geq(N+1) / 2=R+1$. Using $(\star)$, we obtain $d\left(\widetilde{U}_{\theta}, \ell\right) \geq R$. Thus, $d(U, \sigma) \geq R$.

Proof of Proposition 1. Suppose $U$ lies in the block $X_{u}$ and $W$ lies in the block $X_{w}$. We prove the proposition by induction on $m=l(u)+l(w)$.

The base of induction : $m=0$.

The assumption $d(U, W)<R$ implies that $U=W$, because different members of the family $\mathcal{W}^{(i, j)}$ are $N$-disjoint.

The induction step: $m \rightarrow m+1$.

Assuming that the assertion is true whenever $l(u)+l(w) \leq m$, we consider the case where $l(u)+l(w)=m+1$. Without loss of generality, $l(u) \leq l(w)$. Then $l(w) \geq 1$. Thus there exists a boundary plane $\sigma$ in $X_{w}$ such that $l\left(w^{\prime}\right)=l(w)-1$ for the block $X_{w^{\prime}}$ that is glued to $X_{w}$ over $\sigma$.

We have $d(W, \sigma)<R$, because $U$ and $W$ are separated by $\sigma$. Therefore, $W \cap \sigma \neq \varnothing$, because $X_{w}$ and $\sigma$ satisfy the conditions of Lemma4, By Lemma 3, $d(x, W)=d(x, W \cap \sigma)$ for every $x \in U$, whence $d(U, W \cap \sigma)<R$. There is $W^{\prime} \in \mathcal{W}^{(i, j)}, W^{\prime} \subset X_{w^{\prime}}$, such that 
$W^{\prime} \cap \sigma=\sigma \cap W$ by the construction of the family $\mathcal{W}^{(i, j)}$; in particular, $W^{\prime}$ is equivalent to $W$. Then $d\left(W^{\prime}, U\right)<R$, and thus, $W^{\prime}$ is equivalent to $U$ by the inductive hypothesis. Hence, $W$ is equivalent to $U$.

Given $(i, j) \in\{1,2\}^{2}$, for each equivalence class $\widetilde{W}$ of the equivalence relation $\sim_{(i, j)}$ on the family $\mathcal{W}^{(i, j)}$, we consider the set $W:=\bigcup\{U: U \in \widetilde{W}\}$ and denote by $\widetilde{\mathcal{W}}^{(i, j)}$ the family of all such sets. Proposition 1 implies that the covering

$$
\widetilde{\mathcal{W}}=\bigcup\left\{\widetilde{\mathcal{W}}^{(i, j)}:(i, j) \in\{1,2\}^{2}\right\}
$$

of $X$ is 4 -colored and $R$-disjoint. However, $\widetilde{\mathcal{W}}$ is unbounded, since the diameter of each of its members is infinite.

\section{§5. Proof of Theorem 1}

5.1. Extension of the covering members. Let $W$ be a member of the covering $\tilde{\mathcal{W}}$. The sets $W_{u}=X_{u} \cap W, u \in \mathrm{V}(T)$, while nonempty, form the equivalence class $\widetilde{W}$ that corresponds to $W$. We have $W_{u}=U \times V$, where $U \subset \mathrm{H}_{0}$ and $V \subset \mathbb{R}$ are members of the coverings $\tilde{U}^{i}$ and $\tilde{\mathcal{V}}^{j}$ (respectively) for some pair $(i, j) \in\{1,2\} \times\{1,2\}$.

Recall that $U$ is the union of the hexagons labeled by all vertices of the tree $T_{0}$ (see Subsection 3.1 for the definition of $\left.T_{0}\right)$ that lie in a member $U^{\prime}$ of the standard $(N, p)$-covering of $T_{0}$. There is $k \in \mathbb{N} \cup\{0\}$ such that $U^{\prime}$ is contained in the $N$-annulus

$$
A_{k}=\{q: k N \leq|q p| \leq(k+1) N\} \subset T_{0}
$$

centered at $p$.

Now, we construct an extension $\bar{U}^{\prime}$ of $U^{\prime}$ as follows. For each $x \in U^{\prime}$, consider the geodesic segment $\gamma_{x}=x^{\prime} x \subset p x$ of length $N / 2$ (if $|p x|<N / 2$, then $k=0, U^{\prime}=B_{N}(p)$, and we define $\left.\bar{U}^{\prime}:=U^{\prime}\right)$. We put

$$
\bar{U}^{\prime}:=\bigcup\left\{\gamma_{x}: x \in U^{\prime}\right\} \subset T_{0}
$$

and define

$$
\bar{U}:=\bigcup\left\{\theta_{v}: v \in \bar{U}^{\prime} \cap \mathrm{V}\left(T_{0}\right)\right\} \subset \mathrm{H}_{0} .
$$

Note that the set $\bar{U}^{\prime}$ is connected. Indeed, for each $x, y \in \bar{U}^{\prime}$, there exist $x_{1}, y_{1} \in U^{\prime}$ such that $x \in \gamma_{x_{1}}, y \in \gamma_{y_{1}}$. Let $s$ be the center of the tripod $x_{1}, y_{1}, p$. The definition of the set $U^{\prime}$ implies that $|p s| \geq\left(k-\frac{1}{2}\right) N$. There are $x^{\prime} \in p x_{1}$ and $y^{\prime} \in p y_{1}$ such that $\left|p x^{\prime}\right|=\left|p y^{\prime}\right|=k N$. Then $x_{1} x^{\prime}, y_{1} y^{\prime} \subset U^{\prime}$ and either $s \in U^{\prime}$, or $\left|s x^{\prime}\right|,\left|s y^{\prime}\right| \leq N / 2$. In each case $s$ is connected with $x$ and $y$ by paths in $\bar{U}^{\prime}$, so that the set $\bar{U}^{\prime}$ is connected. Then $\bar{U}$ is convex by Remark 3 ,

Recall that $V$ is the union of the intervals labeled by all the vertices of the tree $S_{0}$ (homeomorphic to $\mathbb{R}$; see Subsection 3.1 for the definition of $S_{0}$ ) lying in a member $V^{\prime} \subset S_{0}$ of the standard $(N, d, q)$-covering of $S_{0}$ for some natural $d \leq N$.

We construct an extension $\bar{V}^{\prime}$ of $V^{\prime}$ in a similar way. For each $x \in V^{\prime}$, consider the segment $\gamma_{x}:=x^{\prime} x \subset q x$ of length $N / 2$ (if $|q x|<N / 2$, then either $V^{\prime}=B_{d}(q)$ and we define $\bar{V}^{\prime}:=V^{\prime}$, or $V^{\prime}=B_{d+N}(q) \backslash \operatorname{Int} B_{d}(q)$ and $d<N / 2$, and then we define $\left.\bar{V}^{\prime}:=B_{d+N}(q)\right)$. Consider the set

$$
\bar{V}^{\prime}:=\bigcup\left\{\gamma_{x}: x \in V^{\prime}\right\} \subset S_{0}
$$

and put

$$
\bar{V}:=\bigcup\left\{I_{v}: v \in \bar{V}^{\prime} \cap \mathrm{V}\left(S_{0}\right)\right\} \subset \mathbb{R} .
$$

Since $\bar{V}^{\prime}$ is a segment in $S_{0}$, it is connected. Then $\bar{V}$ is convex by Remark 3 , 
Therefore, $\bar{W}_{u}:=\bar{U} \times \bar{V}$ is a convex subset of $X_{u}$. Moreover, $\bar{W}_{u}$ is compact by construction. We shall call this set the extension of the set $W_{u}$. Note that, as in Lemma 2, the extensions of the sets $W_{u}$ and $W_{w}$ agree for the blocks $X_{u}$ and $X_{w}$ having the common boundary plane $\sigma$; i.e., their intersections with $\sigma$ coincide. The union of the extensions of all block components $W_{u}$ will be called the extension of the set $W$.

We recall the well-known fact that for each point $x$ in a Hadamard space $X$ and for each closed convex subset $A \subset X$ there exists a unique point $p_{x} \in A$ such that $\left|x p_{x}\right|=d(x, A)$ (the point $p_{x}$ is called the metric projection of $x$ to the set $A$ ). The map sending $x \in X$ to its metric projection $p_{x} \in A$ is called the projection to $A$ and is a 1-Lipschitz map.

Proposition 2. For each member $W$ of the covering $\tilde{\mathcal{W}}$, its extension $\bar{W}$ is convex.

Proof. Given $x, y \in \bar{W}$, we prove that the geodesic segment $x y$ lies in $\bar{W}$. Let $X_{u}$, $X_{v} \subset X(u, v \in \mathrm{V}(T))$ be the blocks containing $x$ and $y$, respectively. If $u=v$, then $x, y \in \bar{W}_{u}$, which is convex. Thus, we assume that $u \neq v$. The segment $x y$ intersects consecutively the boundary planes $\sigma_{1}, \ldots, \sigma_{n}$ of the blocks $X_{0}=X_{u}, \ldots, X_{n}=X_{w}$ $\left(\sigma_{i}=X_{i-1} \cap X_{i}\right.$ for each $\left.i \in\{1, \ldots, n\}\right)$ at $z_{i} \in \sigma_{i}, i \in\{1, \ldots, n\}$. The sets $\bar{W}_{i}:=X_{i} \cap \bar{W}$ are convex for all $i \in\{0, \ldots, n\}$. Note that for each $i \in\{1, \ldots, n\}$ the projections of $z_{i}$ to $\bar{W}_{i-1}$ and to $\bar{W}_{i}$ coincide. Indeed, let $t \in \bar{W}_{i}$ be the nearest point to $z_{i}$. By the construction of $\bar{W}_{i}$, the metric projection of $\bar{W}_{i}$ to $\sigma_{i}$ coincides with $\bar{W}_{i} \cap \sigma_{i}$; in particular, the projection $t_{i}$ of $t$ to $\sigma_{i}$ belongs to $\bar{W}_{i}$. Since $\sigma_{i}$ is convex in the Hadamard space $X$, we have $\left|z_{i} t_{i}\right| \leq\left|z_{i} t\right|$. Therefore, the point in $\bar{W}_{i}$ nearest to $z_{i}$ lies in $\bar{W}_{i} \cap \sigma_{i}$. By a similar argument with $\bar{W}_{i-1}$, we show that the projections of $z_{i}$ to $\bar{W}_{i-1}$ and to $\bar{W}_{i}$ coincide. We denote this point by $z_{i}^{\prime}$. By the convexity of $\bar{W}_{i}$, we have $\left|z_{i}^{\prime} z_{i+1}^{\prime}\right| \leq\left|z_{i} z_{i+1}\right|$ for every $i=0, \ldots, n$, where $z_{0}=z_{0}^{\prime}=x, z_{n+1}=z_{n+1}^{\prime}=y$; i.e., the length of the curve $\gamma:=x z_{1}^{\prime} \cup \ldots \cup z_{n}^{\prime} y$ does not exceed that of $x y$, whence $\gamma=x y$. Thus, $x y \subset \bar{W}$.

5.2. Additional colors. Consider the extension $\bar{W}$ of a member $W$ of the covering $\widetilde{\mathcal{W}}$. Note that for each block $X_{u}$ with $X_{u} \cap W \neq \varnothing$, the set $\bar{W}_{u}:=X_{u} \cap \bar{W}$ is convex, compact, and $17 R$-bounded. The last claim follows from the fact that $W_{u}=X_{u} \cap W$ is $15 R$-bounded, and each point $x \in \bar{W}_{u}$ lies at a distance of at most $R$ from $W_{u}$.

Remark 4. For $u, w \in V(T)$, any segment $x y \subset X$ with $x \in W_{u}$ and $y \in W_{w}$ intersects $W_{u^{\prime}}$ if and only if the segment $u w \subset T$ contains $u^{\prime}$.

Put $c=18$. Like the standard coverings of a metric tree (see 92 ), for every member $W$ of the covering $\widetilde{\mathcal{W}}$ we construct a 2 -colored, $R$-disjoint, and $c^{\prime} R$-bounded covering of the extension $\bar{W}$, where $c^{\prime}:=5 c-2$.

Given $x \in X, u \in V(T)$, we write $x \bar{W}_{u}$ for the geodesic segment $x x^{\prime} \subset X$, where $x^{\prime}$ is the metric projection of $x$ to $\bar{W}_{u}$. This notation is consistent, because $\bar{W}_{u}$ is convex, so that $x^{\prime}$ is unique. We fix $v \in \mathrm{V}(T)$ such that $W \cap X_{v} \neq \varnothing$. For every $k \in \mathbb{N} \cup\{0\}$ consider the "annulus"

$$
A_{k}:=\bigcup\left\{\bar{W}_{u}: c k R \leq d\left(\bar{W}_{v}, \bar{W}_{u}\right) \leq c(k+1) R\right\} \subset \bar{W} .
$$

We define a relation $\sim_{k}$ on the set $A_{k}: x \sim_{0} y$ for every $x, y \in A_{0}$, and for each natural $k$, let $x \sim_{k} y$ if and only if there exists a vertex $u \in \mathrm{V}(T)$ such that the geodesic segments $x \bar{W}_{v}$ and $y \bar{W}_{v}$ intersect the set $\bar{W}_{u}$, and

$$
d\left(\bar{W}_{u}, \bar{W}_{v}\right) \geq(c(k-1)+1 / 2) R .
$$

Note that $x \sim_{k} y$ for each $x, y \in \bar{W}_{u} \subset A_{k}$.

We prove that $\sim_{k}$ is an equivalence relation. Indeed, suppose $x \sim_{k} y$ and $y \sim_{k} z$. For any $x \in \bar{W}_{v_{x}}, y \in \bar{W}_{v_{y}}$, and $z \in \bar{W}_{v_{z}}$, there are $u \in \mathrm{V}(T)$ and $w \in \mathrm{V}(T)$ occurring in the 
definition of $\sim_{k}$ for the pairs $\{x, y\}$ and $\{y, z\}$, respectively. Note that $u$ and $w$ lie on the segment $v_{y} v \subset T$. Without loss of generality, suppose that $|u v| \leq|w v|$. Then the segment $z \bar{W}_{v}$ intersects the set $\bar{W}_{u}$; therefore, the points $x$ and $z$ are also equivalent.

Consider the following 2-colored covering of the set $\bar{W}$ : the sets of the first color are all the equivalence classes of $\sim_{k}$ with $k$ even, and the sets of the second color are all the equivalence classes of $\sim_{k}$ with $k$ odd.

We prove that this covering of $\bar{W}$ is $c^{\prime} R$-bounded. Indeed, assume that points $x \in$ $\bar{W}_{v_{x}} \subset A_{k}, y \in \bar{W}_{v_{y}} \subset A_{k}$ are equivalent, and let $u \in \mathrm{V}(T)$ be a vertex such that the segments $x \bar{W}_{v}, y \bar{W}_{v}$ intersect the set $\bar{W}_{u}$ and

$$
d\left(\bar{W}_{u}, \bar{W}_{v}\right) \geq(c(k-1)+1 / 2) R .
$$

For $z, t \in \bar{W}_{u}$ with $z \in x \bar{W}_{v}$, and $t \in y \bar{W}_{v}$, we have $\left|z \bar{W}_{v}\right|,\left|t \bar{W}_{v}\right| \geq(c(k-1)+1 / 2) R$. Then $|x z|=\left|x \bar{W}_{v}\right|-\left|z \bar{W}_{v}\right| \leq\left(2 c-\frac{1}{2}\right) R$, and, similarly, $|y t| \leq\left(2 c-\frac{1}{2}\right) R$. Since $\bar{W}_{u}$ is $(c-1) R$-bounded, we obtain

$$
|x y| \leq|x z|+|z t|+|t y| \leq(5 c-2) R=c^{\prime} R .
$$

Now, we prove that the resulting covering of $\bar{W}$ is $R$-disjoint. Let $U_{1} \subset A_{k}$ and $U_{2} \subset A_{l}$ be sets of the same color.

First, assume that $|k-l| \geq 2$. Without loss of generality, suppose $k \geq l+2$. If $d\left(U_{1}, U_{2}\right)<R$, then $|x y|<R$ for some $x \in \bar{W}_{v_{x}} \subset U_{1}$ and $y \in \bar{W}_{v_{y}} \subset U_{2}$. Note that $d\left(\bar{W}_{v}, \bar{W}_{v_{y}}\right) \leq c(l+1) R$, whence $|z t| \leq c(l+1) R$ for some $z \in \bar{W}_{v_{y}}, t \in \bar{W}_{v}$. Then

$$
|x t| \leq|x y|+|y z|+|z t|<R+(c-1) R+c(l+1) R=c(l+2) R \leq c k R .
$$

This contradicts the inequality $c k R \leq d\left(\bar{W}_{v}, \bar{W}_{v_{x}}\right)$.

Now we assume that $k=l, \bar{W}_{a} \subset U_{1}$, and $\bar{W}_{b} \subset U_{2}$. It suffices to prove that $d\left(\bar{W}_{a}, \bar{W}_{b}\right) \geq R$. Let $u \in \mathrm{V}(T)$ be the center of the tripod $a b v \subset T$. Then any geodesic segment going from $\bar{W}_{a}$ or $\bar{W}_{b}$ to $\bar{W}_{v}$ passes through $\bar{W}_{u}$, and

$$
d\left(\bar{W}_{u}, \bar{W}_{v}\right)<(c(k-1)+1 / 2) R .
$$

We show that $d\left(\bar{W}_{a}, \bar{W}_{u}\right) \geq R / 2$. Indeed, suppose this is not true. Then there are $x_{1} \in \bar{W}_{a}, x_{2}, z_{2} \in \bar{W}_{u}, z_{1} \in \bar{W}_{v}$ such that $\left|x_{1} x_{2}\right|<R / 2,\left|z_{1} z_{2}\right|<(c(k-1)+1 / 2) R$. Thus,

$$
\left|x_{1} z_{1}\right| \leq\left|x_{1} x_{2}\right|+\left|x_{2} z_{2}\right|+\left|z_{2} z_{1}\right|<R / 2+(c-1) R+(c(k-1)+1 / 2) R=c k R,
$$

which contradicts the inclusion $\bar{W}_{a} \subset A_{k}$.

Similarly, $d\left(\bar{W}_{b}, \bar{W}_{u}\right) \geq R / 2$. Since $u \in a b \subset T$, we have

$$
d\left(\bar{W}_{a}, \bar{W}_{b}\right) \geq d\left(\bar{W}_{a}, \bar{W}_{u}\right)+d\left(\bar{W}_{u}, \bar{W}_{b}\right) \geq R .
$$

We restrict the constructed covering of $\bar{W}$ to $W \subset \bar{W}$; i.e., for each member of the covering we consider its intersection with $W$. The new covering of $W$ is again 2-colored, $R$-disjoint, and $c^{\prime} R$-bounded with $c^{\prime}=5 c-2=88$. We denote this covering by $X_{W}$, $x_{W}=X_{W}^{1} \cup X_{W}^{2}$.

For each triple of numbers $(i, j, k) \in\{1,2\}^{3}$, consider the following 8-colored covering of $X$ :

$$
X:=\bigcup\left\{X_{W}^{k}: W \in \tilde{\mathcal{W}}^{(i, j)},(i, j, k) \in\{1,2\}^{3}\right\} .
$$

The covering $X$ is $c^{\prime} R$-bounded because so are the families $X_{W}^{k}, k=1,2$. We prove that $X$ is $R$-disjoint. Indeed, let $U \in X_{W_{1}}^{k}, V \in X_{W_{2}}^{k}$ be two different sets of the same color for some triple of numbers $(i, j, k) \in\{1,2\}^{3}$, where $W_{1}, W_{2} \in \widetilde{\mathcal{W}}^{(i, j)}$. If $W_{1} \neq W_{2}$, then $d(U, V) \geq d\left(W_{1}, W_{2}\right) \geq R$, because $U \subset W_{1}, V \subset W_{2}$, and the families $\tilde{\mathcal{W}}^{(i, j)}$ are $R$-disjoint. If $W_{1}=W_{2}$, then $d(U, V) \geq R$ because $X_{W_{1}}^{k}$ is $R$-disjoint. This completes the proof of Theorem 1 . 


\section{§6. Applications}

We recall some definitions.

Definition 3 (Assouad [1]). The Assouad-Nagata dimension $\operatorname{dim}_{\mathrm{AN}} X$ of a metric space $X$ is the smallest integer $n$ such that for each $R>0$ there exists an $(n+1)$-colored, $R$-disjoint, and $C R$-bounded covering of $X$, where the number $C>0$ is independent of $R$.

Definition 4 (Buyalo [6]). The linearly controlled dimension $\ell$-dim $X$ of a metric space $X$ is the smallest integer $n$ such that for each $R \leq 1$ there exists an $(n+1)$-colored, $R$-disjoint, and $C R$-bounded covering of the space $X$, where the number $C>0$ is independent of $R$.

It is easily seen that $\operatorname{dim}_{\mathrm{AN}} X=\max \{\ell-\operatorname{dim} X, \ell$-asdim $X\}$.

Recall that $\widetilde{M}$ is the universal cover of the graph-manifold $M$. A remark to [9, Proposition 2.7] implies that $\ell-\operatorname{dim} \widetilde{M}=\operatorname{dim} \widetilde{M}$, whence $3=\operatorname{dim} \widetilde{M} \leq \operatorname{dim}_{\mathrm{AN}} \widetilde{M}$. Theorem 1 shows that $\operatorname{dim}_{\mathrm{AN}} \widetilde{M} \leq 7$, which gives Corollary 1. However, by the Bell-Dranishnikov result (see [3]), asdim $\widetilde{M}=3$, which supports the following conjecture.

Conjecture. Let $\widetilde{M}$ be the universal cover of a graph-manifold. Then $\operatorname{dim}_{\mathrm{AN}} \widetilde{M}=3$.

Corollary 2 follows from Corollary 1 and 9 , Theorem 1.3].

Definition 5. A metric space $X$ is said to be Lipschitz $n$-connected, $n \geq 0$, if for each $m \in\{0, \ldots, n\}$ there exists $\gamma>0$ such that every $\lambda$-Lipschitz map $f: S^{m} \rightarrow X$ admits a $\gamma \lambda$-Lipschitz extension $\bar{f}: B^{m+1} \rightarrow X$, where $S^{m}$ and $B^{m+1}$ are the unit sphere and the unit disk in $\mathbb{R}^{m+1}$, respectively.

In 4 it was proved that the Hadamard spaces are Lipschitz $n$-connected for each $n \geq 0$. Thus, the model space $X$ is Lipschitz $n$-connected for each $n \geq 0$. By Corollary 1, the space $X$ has finite Assouad-Nagata dimension. Now, Corollary 3 follows from [9. Theorem 1.5].

\section{REFERENCES}

[1] P. Assouad, Sur la distance de Nagata, C. R. Acad. Sci. Paris Sér. I Math. 294 (1982), no. 1, 31-34. MR0651069 (83b:54034)

[2] J. A. Behrstock and W. D. Neumann, Quasi-isometric classification of graph manifold groups, Duke Math. J. 141 (2008), no. 2, 217-240. MR2376814 (2009c:20070)

[3] G. Bell and A. Dranishnikov, On asymptotic dimension of groups acting on trees, Geom. Dedicata 103 (2004), no. 1, 89-101. MR.2034954(2005b:20078)

[4] M. R. Bridson and A. Haefliger, Metric spaces of nonpositive curvature, Grundlehren Math. Wiss., Bd. 319, Springer-Verlag, Berlin, 1999. MR.1744486 (2000k:53038)

[5] D. Yu. Burago, Yu. D. Burago, and S. V. Ivanov, A course of metric geometry, Inst. Kompyuter. Issled., Moscow-Izhevsk, 2005. (Russian)

[6] S. V. Buyalo, Asymptotic dimension of a hyperbolic space and the capacity dimension of its boundary at infinity, Algebra i Analiz 17 (2005), no. 2, 70-95; English transl., St. Petersburg Math. J. 17 (2006), no. 2, 267-283. MR2159584 (2006d:31009)

[7] A. Dranishnikov and M. Zarichnyi, Universal spaces for asymptotic dimension, Topology Appl. 140 (2004), no. 2-3, 203-225. MR.2074917(2005e:54032)

[8] M. Gromov, Asymptotic invariants of infinite groups, Geometric Group Theory, Vol. 2 (Sussex, 1991), London Math. Soc. Lecture Note Ser., vol. 182, Cambridge Univ. Press, Cambridge, 1993, pp. 1-295. MR $1253544(95 \mathrm{~m}: 20041)$

[9] U. Lang and T. Schlichenmaier, Nagata dimension, quasisymmetric embeddings, and Lipschitz extensions, Int. Math. Res. Not. 2005, no. 58, 3625-3655. MR2200122 (2006m:53061) 
[10] J. Roe, Lectures on coarse geometry, Univ. Lecture Ser., vol. 31, Amer. Math. Soc., Providence, RI, 2003. MR2007488 (2004g:53050)

[11] G. Yu, The Novikov conjecture for groups with finite asymptotic dimension, Ann. of Math. (2) 147 (1998), no. 2, 325-355. MR.1626745 (99k:57072)

Mathematics and Mechanics Department, St. Petersburg State University, 28 UniversitetSkit Prospekt, Peterhoff, St. Petersburg 198504, Russia

E-mail address: alvismi@gmail.com

Received 23/APR/2009

Translated by THE AUTHOR 